\title{
Wireless, In Vivo Neural Recording using a Custom Integrated Bioamplifier and the Pico System
}

\author{
David Cheney, Aik Goh, Jie Xu, Karl Gugel, Member IEEE, John G. Harris, Member IEEE, \\ Justin C. Sanchez, Member IEEE, and Jose C. Principe, Fellow IEEE
}

\begin{abstract}
This paper describes a wireless system for sampling multiple channels of neural activity based on a low-power, custom 80dB-gain integrated bioamplifier, Texas Instrument's MSP430 microprocessors, and Nordic Semiconductor's ultra low power, high bandwidth RF transmitter/receivers. The system's features are presented as well as results of spike potentials from a live subject.
\end{abstract}

\section{INTRODUCTION}

$\mathrm{T}$ HE development of translational neuroprosthetics to aid patients who suffer from neurological disorders hinges on the long-term goal of providing a direct interface for neural rehabilitation, communication, and control over a period of years. Without the assurance that the neural interface will yield reliable recording, preprocessing, and neural encoding/decoding in a small portable (or implantable) package over the lifetime of the patient, it is likely the current advances in brain-machine interfaces will only be available for limited clinical practice. Because of the immediate clinical needs, several groups have focused on acquiring the knowledge and leveraging current technology to achieve improved wireless, portable neural electrode interfaces to record from many neurons over extended periods of time [1-3]. However, the major bottlenecks in the designs are the power consumption and high data rate transmission requirements from an implanted unit to an external device. Data bandwidths can typically exceed $1 \mathrm{Mb} / \mathrm{s}$ when monitoring neural signal activity from multiple electrodes and as a result, many of the systems with wireless links are not fully implantable and require large backpacks [4-6].

At the University of Florida, we are pursuing multiple paths [7-12] to ultimately achieve a fully implantable wireless neural recording interface. In the particular path described here, the previously reported Pico Recording system [10] is capable of replacing a multi-conductor commutator cable used in animal experimental paradigms with a high-bandwidth, low-power wireless interface, which allows the subject more mobility and a more natural response to a given experiment. This new wireless interface presented a compact architecture as well as new challenges related to the power consumption of the remote device.

Manuscript received February 15, 2007. This work was supported by the U.S. Defense Advanced Research Projects Agency (DARPA), project number 00027668, Computational Engines and Hybrid VLSI Chips for Multi-channel Neural Data Analysis.

David Cheney, Aik Goh, Jie Xu, Karl Gugel, John G. Harris, and Jose C. Principe are with the Department of Electrical and Computer Engineering at the University of Florida, Gainesville, FL 32611 USA (email: [djcheney, gohak, jiexu,gugel]@ufl.edu, [harris, principe]@cnel.ufl.edu).

Justin C. Sanchez is with the Department of Pediatrics, Division of Neurology, University of Florida, Gainesville, FL 32611 USA (email: jcs77@ufl.edu).
In the next step of the design of the Pico Neural Recording system, we present our approach to local data processing and wireless data transmission.

To optimize the trade-off between power consumption and processing power, a low-power, low-noise, high-gain amplifier was designed in conjunction with off-the-shelf components, specifically MSP430F1611 as the microcontroller and the Nordic nRF2401 wireless transceiver. The processor consumes less than two milli-Watts of power with 8 MIPs of processing and has the added benefit of conserving space by integrating two UART's, three DMA channels, and an eight-channel 12-bit A/D converter all in one small package. In this work, we demonstrate the integration of the low-power amplifier with the Pico system and collect data from a live subject.

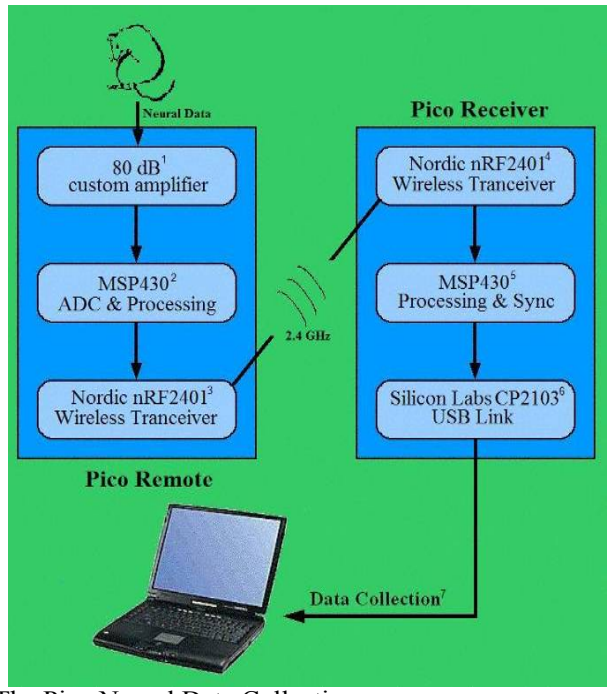

Fig. 1. The Pico Neural Data Collection

\section{FUNCTIONAL DESCRIPTION}

The Pico Neural Data Collection System consists of the Pico Remote (battery-powered neural amplifiers, a neural data processor, and a wireless transceiver), the Pico Receiver (a wireless-transceiver to USB converter), and PC-based software (for spike sorting and firing rate quantification). Fig. 1 provides a block diagram for signal flow throughout the system. The Pico Remote senses single- and multiunit neuronal activity from 16 chronically implanted microelectrodes from a laboratory rat. The signals are amplified $(80 \mathrm{~dB})$ with a custom amplifier and fed to one of eight 12-bit $\mathrm{A} / \mathrm{D}$ channels sampling at $20 \mathrm{kHz}$ on the MSP430. The MSP430 has two modes: spike sorting and spike reading. While in the spike sorting mode, raw sampled data from a single probe are sent over the Nordic transceiver for reception by the Pico Receiver. This mode allows the user to analyze in detail the raw recording from the microelectrodes to set thresholds for detecting and sorting spikes (action potentials). In the spike reading mode, the 
MSP430 executes the spike detection through user defined thresholds and sorting. The firing rate for each neuron is computed locally and sent over the Nordic transceiver for reception by the Pico Receiver.

The Nordic transceiver is capable of up to $250 \mathrm{kbit} / \mathrm{sec}$ sustained data transfer of packet data. Data sent over the Nordic transceivers are fed to another MSP430 processor for buffering and formatting and for the eventual pass through to a Silicon Laboratories CP2103 USB bridge to software running on a PC.

\section{A. The Amplifier}

The custom amplifier consists of two stages: a low-noise $40 \mathrm{~dB}$ preamplifier and a second-stage $40 \mathrm{~dB}$ amplifier which were fabricated in the $0.6 \mathrm{um}$ AMI CMOS process. Fig. 3 shows the schematic of the preamplifier and the second stage is in fig. 4. The amplifier designs are based on the work of Reid Harrison [11]

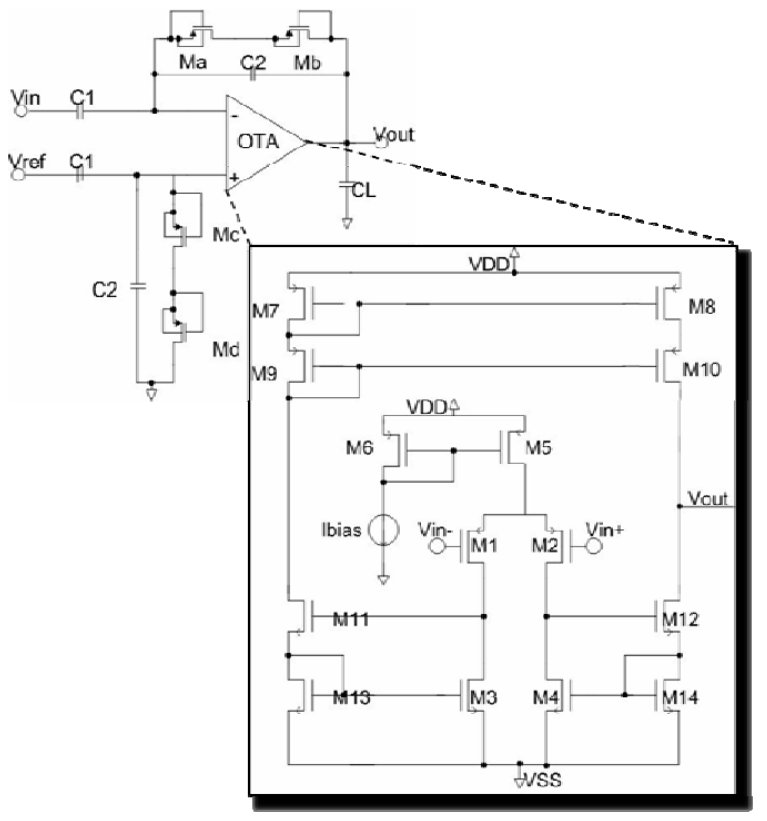

Fig. 3. First stage Amplifier

The first stage midband gain, $A_{M}$, is $-C_{1} / C_{2}$, the lower corner frequency is at $\omega_{1} \approx 1 /\left(\mathrm{RC}_{2}\right)$, and the higher corner frequency is at $\omega_{2} \approx \mathrm{g}_{\mathrm{m}} \mathrm{C}_{2} /\left(\mathrm{C}_{\mathrm{L}} \mathrm{C}_{1}\right)$, where $\mathrm{g}_{\mathrm{m}}$ is the transconductance of the operational transconductance amplifier (OTA) shown in the lower portion of fig. 3 [12]. In order to get a low cut-off frequency, a large resistance is needed in the feedback loop provided by two diodeconnected transistors, $\mathrm{Ma}$ and $\mathrm{Mb}$, acting as "pseudo resistors" without sacrificing die area.

TABLE I

AMPLIFIER SPECIFICATIONS

\begin{tabular}{lll}
\hline \hline \multicolumn{1}{c}{ Parameter } & Preamplifier & Second-stage amplifier \\
\hline Supply voltage & $5 \mathrm{~V}$ & $5 \mathrm{~V}$ \\
Power consumption & $80 \mathrm{uW}$ & $\sim 120 \mathrm{uW}$ \\
Gain & $39.46 \mathrm{~dB}$ & $\sim 40 \mathrm{~dB}$ \\
Bandwidth & $5.4 \mathrm{kHz}$ & $19 \mathrm{kHz}$ \\
Lower cutoff frequency & $\sim 0.3 \mathrm{~Hz}$ & $<1 \mathrm{~Hz}$ \\
Die area & $91800 \mathrm{um}^{2}$ & $66400 \mathrm{um}^{2}$ \\
Input referred noise & $5.94 \mathrm{uVrms}$ & Not available \\
\hline \hline
\end{tabular}

Since noise performance is important in biological preamplifier designs, it is minimized by carefully choosing the width and length of the transistors. However, there is a trade-off between stability and low noise. Since low noise is critical in this application, decreasing the transconductance of M1, M2, M7, M8 and the four transistors of the two Wilson current mirrors can minimize the thermal noise. Flicker noise, which is important in low frequency applications, is minimized by increasing the device area. Because PMOS devices exhibit less flicker noise than NMOS, PMOS is used in the input pair.

Since the typical neural signal amplitude is on the order of tens to hundreds of microvolts, the output voltage of the preamplifier is still too small for signal processing by an analog to digital converter. Therefore, a second-stage amplifier is required [13], in which $C_{1} / C_{3}$ determines the mid-band gain, while Vref and diode-connected resistors provide the dc operational bias. The OTA is a typical two stage OTA with a P-type input differential pair loaded with Wilson current mirrors followed by a class A output stage.

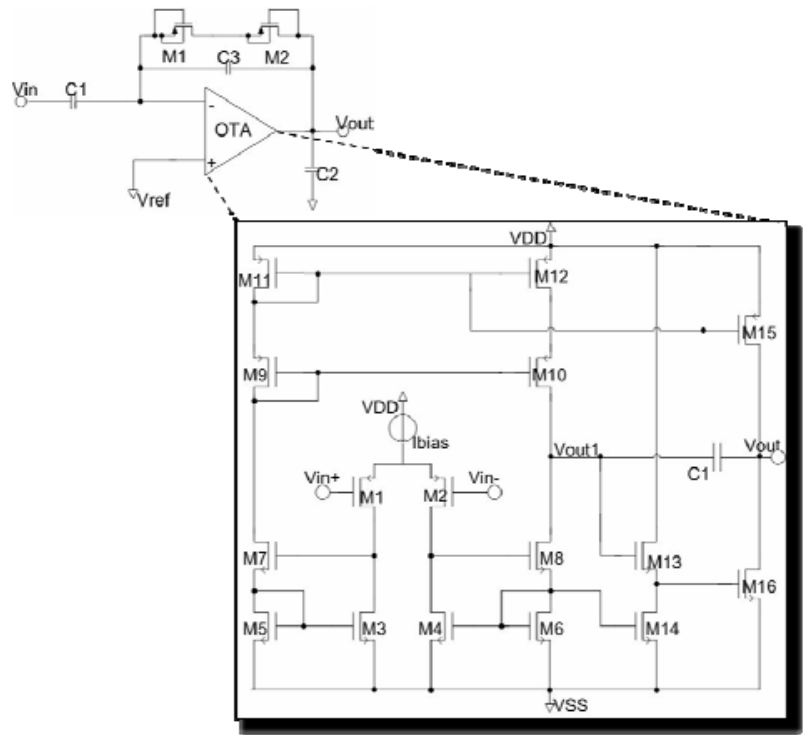

Fig. 4. Second Stage Amplifier

\section{B. The Processor}

The MSP430F1611 processor samples each analog channel at $20 \mathrm{kHz}$ with an onboard 12-bit A/D converter. Depending on the mode in which the processor is functioning, the samples are handled differently.

While in the spike sorting mode, no signal processing occurs and the raw samples are sent directly to the transceiver for processing by software running on the PC. The data are sent as 12-bit samples at rate of $20 \mathrm{k}$ samples/second for a data rate of $240 \mathrm{k}$ samples/second.

In the spike counting mode, each sample is compared against thresholds that define a spike using well established spike-sorting techniques. If all the threshold conditions are met, a counter is incremented. Each probe can have as many as three rate counters corresponding to three neurons per electrode. Every $100 \mathrm{~ms}$ the bin counts are sent to the wireless transceiver for processing on a PC.

\section{The Pico Receiver}

On the receiving side of the RF link data are collected via 
a Nordic nRF2401 transceiver on the Pico Receiver. The RF link is controlled by another MSP430F1611 microcontroller and results are passed on to a PC via a USB bridge (CP2103 from Silicon Laboratory). The USB bridge is compliant with the USB 2.0 specification and has a maximum throughput of $12 \mathrm{Mbits} / \mathrm{sec}$.

\section{User Software}

The Pico Remote is configured with software running on Windows-based PC's. The testing software has three separate applications: Spike Capture, Spike Detection Parameter Initialization, and Binned Spike Data (firing rate) Display.

The Spike Capture application is a Matlab GUI that captures and displays raw sample data sent from the Pico Remote. This application does not configure the Pico Remote but is used solely for viewing and storing a single channel's raw sample data for analysis.

The Spike Detection Parameter Initialization application is in development and will accommodate configuring three counters for each probe. Fig. 5 shows screenshots of the user interface.

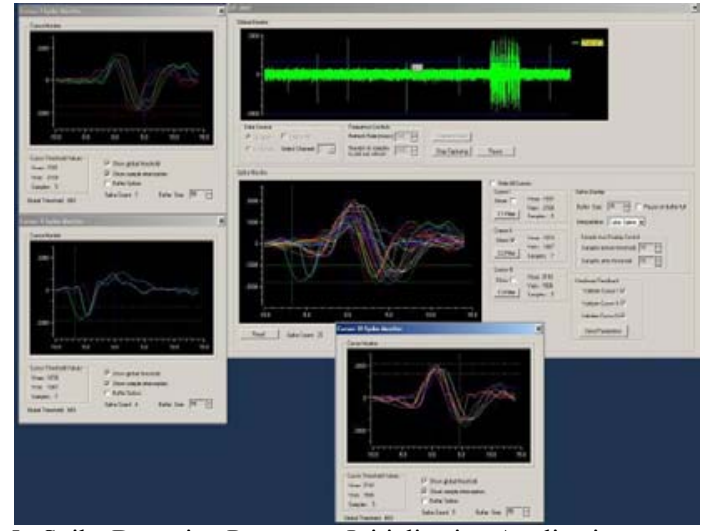

Fig. 5. Spike Detection Parameter Initialization Application

The Spike Rate Data Display application is used for demonstration purposes. In an actual lab environment, a windows' service reads the spike counts from the USB port and stores them in shared memory so any windows' application can process them.

\section{IN VIVO TESTING AND RESULTS}

Since invasive microwire recording technologies are targeting single unit neuronal activity, we will use the action potential (spike) as our standard for evaluation of data collected from the neural probes [14]. The Pico system and custom amplifier have been previously tested extensively using a Bionic Neural Simulator to generate stereotypical physiologic signals on the electronics workbench.

In vivo tests used Adult male $250 \mathrm{~g}$ Sprague-Dauley rats to benchmark the recording performance of the custom amplifier and Pico system. All procedures have been approved by the University of Florida IACUC Board. A 16 electrode $(2 \times 8)$ microwire array was implanted to a depth of $1.66 \mathrm{~mm}$ into the forelimb region of the primary motor cortex. The array was then grounded using a 1/16" diameter stainless steel screw implanted in the skull.

Fig. 6 graphs a 30 second trace of neural recordings captured with the Pico system and gives a global perspective into the peak-to-peak amplitudes and occurrences of spiking neurons. The average of the data over the thirty seconds is expected to be around zero. Instead, there is an offset of nearly $9 \mathrm{uV}$, which has been determined to be attributed to not precisely biasing the amplifiers.

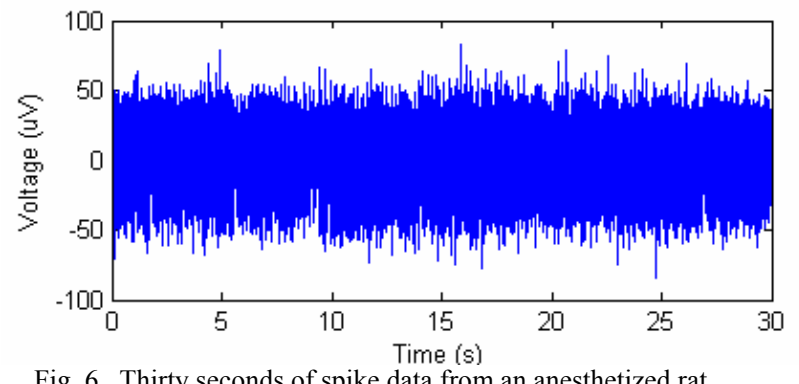

Fig. 6. Thirty seconds of spike data from an anesthetized rat.

In fig. 7, we zoom in on two individual action potentials where the range of the noise floor can take values of $\pm 25 \mu \mathrm{V}$ with the spikes reaching $-75 \mu \mathrm{V}$.

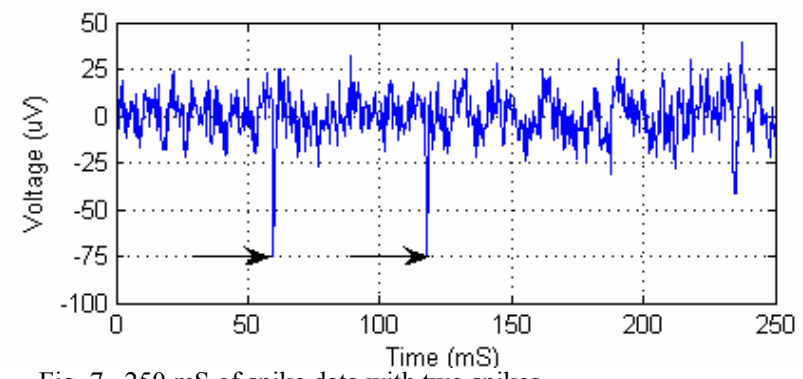

Fig. 7. $250 \mathrm{mS}$ of spike data with two spikes

Science Products' Spike 2 analysis software was used to extract neural data from the dataset. Fig. 8 shows the average waveshape of two neurons firing on the probe from which we sampled.
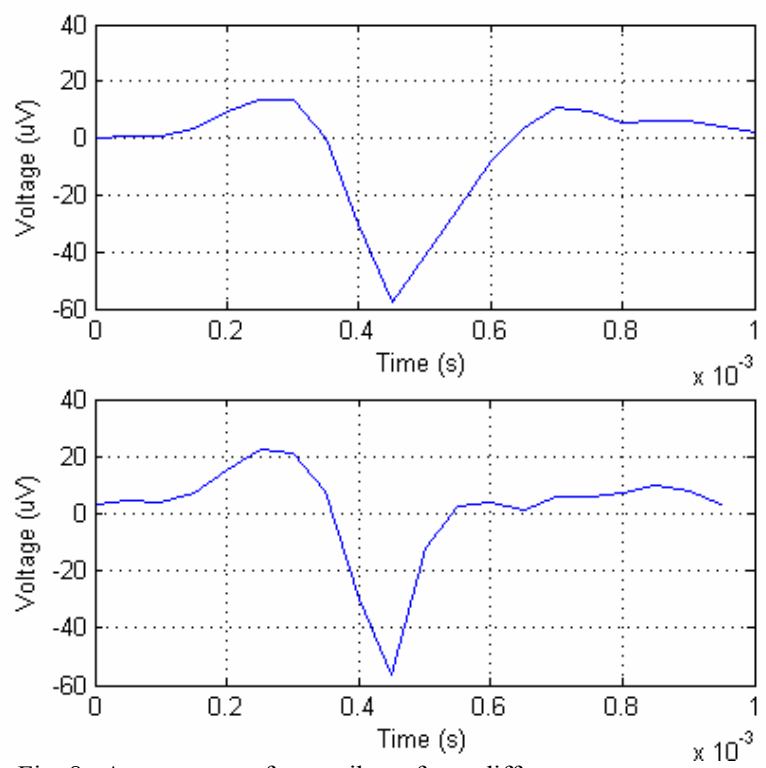

Fig. 8. Average waveform spikes of two different neurons

It is difficult to calculate meaningful signal-to-noise ratios (SNR) on neurophysiologic data, since the spike signal is not continuous like those used in traditional communication's calculations [15]. Table II presents an attempt to derive meaningful SNR calculations from the 
average spike value. The RMS voltage of the noise floor was calculated by taking the square root of the average of the square of samples in the noise floor. The samples used in the calculation were extracted by looking for periods of data where no spikes appeared.

TABLE II

SNR CALCULATIONS

\begin{tabular}{rrr}
\hline & Neuron 1 & \multicolumn{1}{c}{ Neuron 2 } \\
\hline Noise $\mathrm{V}_{\mathrm{rms}}$ & $8.24 \mu \mathrm{V}$ & $8.24 \mu \mathrm{V}$ \\
Neuron $\mathrm{V}_{\mathrm{pp}}$ & $71.13 \mu \mathrm{V}$ & $79.33 \mu \mathrm{V}$ \\
$\mathrm{SNR}$ & $18.72 \mathrm{~dB}$ & $19.67 \mathrm{~dB}$ \\
\hline \hline
\end{tabular}

To provide a baseline for quantifying the noise attributed by the system, the inputs to the probes were tied to the reference and the corresponding trace is given in fig. 9. These results suggest the source of the dc offset is inherent in the system, which supports the need to carefully adjust the bias of the amplifiers. When these data are normalized the RMS voltage of the noise using the same process described earlier, yields $0.66 \mu \mathrm{V}$, much lower when compared to the $8.24 \mathrm{uV}$ in the actual test.

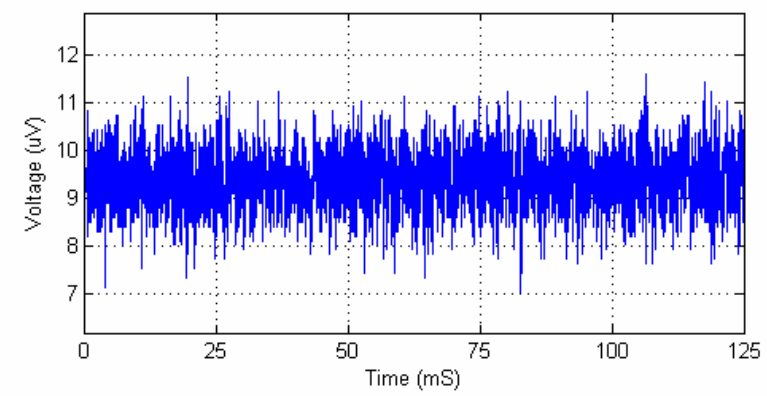

Fig. 9. Noise introduced by the Pico system

For all of the neural recordings presented here, the system power consumption for the Pico Remote is quantified in Table III. The Pico remote consumes around $130 \mathrm{~mW}$ while bin counting, which on a $3.7 \mathrm{~V}, 150 \mathrm{mAh}$, LiON battery would provide about 4.25 hours of operation.

TABLE III

PICO REMOTE POWER CONSUMPTION

\begin{tabular}{lll}
\hline \hline \multicolumn{1}{c}{ System } & \multicolumn{1}{c}{ Spike Sorting } & Bin Counting \\
\hline $\begin{array}{l}\text { Pico Remote } \\
\begin{array}{c}\text { Pico Remote } \\
\text { with TDT Headstage }\end{array}\end{array}$ & $100 \mathrm{~mW}$ & $87 \mathrm{~mW}$ \\
\hline \hline
\end{tabular}

\section{CONCLUSIONS \& FUTURE WORK}

Now that the initial goal of bringing actual neural data from a live subject over the wireless link to a PC has been achieved, we can now move forward in processing the data on the Pico remote by applying our spike counting algorithm. There are still additional tests that have to be completed, such as, comparing in a parallel test, raw signal data and spike counts with a commercial system, such as those from TDT Systems.

To ensure data integrity over the wireless link, we have created specifications for a robust communications protocol that utilizes packet acknowledgements and resends. Any data sent over the wireless network will be acknowledged by the receiving device. If the sending device does not receive an acknowledgement in a few milliseconds, the data are resent. A new version of the Nordic part allows for data resends in hardware reducing power and firmware complexity. Once the latest Nordic parts and the protocol are implemented, we expect data loss to be very close to zero.

Once the tests are completed and the design is stable, the system will be repackaged in a small rat backpack not larger than 1.5 " $\mathrm{x} 3$ " in size and weighing a few ounces.

\section{ACKNOWLEDGMENT}

The authors would like to thank Du Chen and Yuan Li for their contributions to the amplifier design.

\section{REFERENCES}

[1] T. Akin, B. Ziaie, S. A. Nikles, and K. Najafi, "A modular micromachined high-density connector system for biomedical applications," IEEE Transactions on Biomedical Engineering, vol. 46, pp. 471-480, 1999.

[2] N. A. Blum, B. G. Carkhuff, H. K. J. Charles, R. L. Edwards, and R. A. I. T. B. E.-J. H. U. t. f. A. Meyer, "Multisite microprobes for neural recordings," IEEE Transactions on Biomedical Engineering, vol. 38, pp. $68-74,1991$.

[3] C. I. Palmer, "Long-Term Recordings In The Cat Motor Cortex - UnitActivity And Field Potentials From Sensory And Brain-Stem Stimulation As A Means Of Identifying Electrode Position," Journal Of Neuroscience Methods, vol. 31, pp. 163-181, 1990.

[4] G. Buzsaki, "Multisite recording of brain field potentials and unit activity in freely moving rats," Journal of Neuroscience Methods, vol. 28, pp. 209-217, 1989.

[5] K. D. Wise, D. J. Anderson, J. F. Hetke, D. R. Kipke, and K. Najafi, "Wireless implantable microsystems: High-density electronic interfaces to the nervous system," Proceedings Of The IEEE, vol. 92, pp. 76-97, 2004.

[6] P. Grohrock, U. Hausler, and U. Jurgens, "Dual-channel telemetry system for recording vocalization-correlated neuronal activity in freely moving squirrel monkeys," Journal Of Neuroscience Methods, vol. 76, pp. 7-13, 1997.

[7] R. Bashirullah, J. G. Harris, J. C. Sanchez, T. Nishida, J. C. Principe "Florida wireless implantable recording electrodes (FWIRE) for brain machine interfaces," IEEE Symposium on Circuits and Systems, New Orleans, 2007.

[8] C. L. Rogers, J. G. Harris, J. C. Principe, J. C. Sanchez, "A pulsebased feature extractor for spike sorting neural signals," IEEE Symposium on Circuits and Systems, New Orleans, 2007.

[9] E. Patrick, M. Ordonez, N. Alba, J. C. Sanchez, and T. Nishida, "Design and Fabrication of a Flexible Substrate Microelectrode Array for Brain Machine Interfaces," IEEE International Conference of the Engineering in Medicine and Biology Society, 2006.

[10] G. Cieslewski, D. Cheney, K. Gugel, J. Sanchez, and J Principe, "Neural Signal Sampling via the Low Power Wireless Pico System.", Intl. Conf. of Engineering in Medicine and Biology Society, pp. 59045907, Aug. 2006.

[11] R.R. Harrison and C. Charles, A low-power low-noise CMOS amplifier for neural recording applications, IEEE Journal of SolidState Circuits, 38: 958-965, June 2003.

[12] D. Chen, An Ultra-low Power Neural Recording System Using Pulse Representations, Ph.D. dissertation, Department of Electrical and Computer Engineering, University of Florida, Gainesville, FL, May 2006.

[13] Y. Li, An Integrated Multichannel Neural Recording System With Spike Outputs, Ph.D. dissertation, Department of Electrical and Computer Engineering, University of Florida, Gainesville, FL, Dec 2006.

[14] M. A. L. Nicolelis, Methods for Neural Ensemble Recordings. Boca Raton: CRC Press, 1999.

[15] Z. Nenadic, J. Burdick, "Spike Detection Using the Continuous Wavelet transform", IEEE Transactions on Biomedical Engineering, vol. 52, no.1, pp. 74-87, January 2005. 